\title{
Diffractive Performance of Square Fresnel Zone Plates
}

\author{
Javier Alda ${ }^{1}$, José María Rico-García ${ }^{2}$, Francisco Javier Salgado-Remacha ${ }^{2}$ \\ Luis Miguel Sanchez-Brea \\ Applied Optics Complutense Group, Optics Department, \\ University Complutense of Madrid \\ ${ }^{1}$ School of Optics. Ave. Arcos de Jalón, 118. 28037 Madrid (Spain) \\ ${ }^{2}$ Facultad de Ciencias Físicas, Ciudad Universitaria s.n., 28040, Madrid (Spain) \\ E-mail corresponding author: j.alda@opt.ucm.es
}

\begin{abstract}
We analyze the optical behavior of Square Fresnel Zones Plates. A theoretical analysis and numerical simulations based on the Rayleigh-Sommerfeld approach have been developed analyzing properties such as the depth of focus and the intensity of the focus in terms of the number of zones. In addition, an experimental verification has been performed using a Spatial Light Modulator to implement the designed Square Fresnel Zones Plates.
\end{abstract}

PACS: 42.25.Fx Diffraction and scattering, 42.79.-e Optical elements, devices, and systems, 42.79.Bh Lenses, prisms and mirrors, 


\section{Introduction}

Diffractive optics is frequently used in micro-optics design. The basic foundation of a diffractive lens is the zoning of the aperture of the lens that follows a customized pattern. This pattern is based on the Fresnel zone concept [1]. The Fresnel Zone Plate (FZP) is typically realized onto a flat surface, although some refined designs can be adapted to three dimensional geometries [2]. The typical flat arrangement is well suited for the integration of diffractive lenses within a micro-optics set-up [3],[4]. The most common FZP designs have circular shape zones, although they can be modified to include conic shapes for astigmatism and off-axis operation [2]. These zones are also known as semi-periodic zones because the phase of the total amplitude arriving to the image point from the location of a given zone is shifted in $\pi$ with respect to the phase coming from the adjacent zones. This phase can be properly compensated by an appropriate amplitude and phase mask. The number of phase levels of such a mask would be only two. When improving the performance of these elements a multi-level phase mask is proposed to define new sub-zones having an adjacent phase shift of $\pi / 2, \pi / 3$, etc. The number of phase levels grows with the successive sub-zoning. A typical solution for a multilevel phase mask uses $2^{n}$. This is done to allow an easier fabrication using photolithographic techniques [5]. Finally, a kinoform [6] is obtained when allowing a continuous phase level along the aperture of the lens producing a surface relief profile [7]. In some designs, circular symmetry is not used, as those based on hyperbolic contours or shapes. These designs show interesting properties and allow new uses [8][9][10]. Also a square FZP has been proposed as a combination of cylindrical FZP [11]. Both the orthogonal superposition of cylindrical FZPs and the hyperbolic FZP may show a focalization pattern with cross-like irradiance distributions. Polygonal Fresnel zones can be designed by substituting the circular shape of the Fresnel zones by a polygonal one [12]. The simplest 
polygonal shape is a triangle, although the square, due to its natural fitting to the rectangular coordinate system, has deserved special attention since long ago [13], and also recently [14]. Some ideas have been proposed for the optimization of the zoning of the square FZP in order to improve its performance [12],[15]. The zones obtained using polygonal boundaries are an approximation to the actual Fresnel zones and should be named as pseudo-Fresnel zone. However, for the sake of convenience we will still name them as square, or polygonal, Fresnel zones. The optimization procedure used to build a square Fresnel zone has produced an interesting outcome: the phase levels associated with the successive zones follow a regular pattern of only 5 values plus the phase level corresponding to the central zone [12]. Then, in this work we analyze and realize a new Square Fresnel Zone Plate (SFZP) that has an intrinsic rectangular geometry and can be fabricated with only 6 phase levels. There are several situations where this approach can be useful. For example, the development of IR reflectarrays is based on the phase induced by resonant subwavelength elements [16]. These induced phases are depending on the spatial dimensions of the individual resonant elements. Having a low number of phase levels, and therefore a low number of geometric parameters, would help the design and fabrication of these devices. Moreover, in those applications where the pixel size and the rectangular geometry of the pixel's arrangement are of importance, this design would be more robust to fabrication imperfections.

In section 2 of this manuscript we present and explain in more detail the basic design parameters of the SFZP. The irradiance around the focus produced by this diffractive element is computed throughout a Rayleigh-Sommerfeld model in section 3. The numerical results predict the expected behavior of these novel diffractive elements. Section 4 describes the experimental set-up employed to realize a working SFZP. A Spatial Light Modulator (SLM) is used to implement the SFZP. The SLM allows to realize a given phase level without the restriction of the $2^{\mathrm{n}}$ level associated with the microlithography fabrication techniques with 
low values of $n$. This flexibility in phase is counterweighted by the restriction on the location of the square contours and its fitting with the actual geometry of the SLM pixels. The results obtained from the experiment are compared with those coming from the simulation. The overall results are in good agreement between the numerical results and the experimental data and the discrepancies are properly analyzed. Finally, section 5 presents the main conclusions derived from this study.

\section{Square Fresnel Zones}

FZP are typically arranged as having a circular shape. The values of their radii, $r_{m}$, are given as a function of the wavelength within the medium, $\lambda$, the desired focal length, $f^{\prime}$, and the order of the zone, $m$. The basic design relation is

$$
r_{m}=\sqrt{f^{\prime} \lambda m+\left(\frac{\lambda m}{2}\right)^{2}},
$$

where the second term within the square root is usually neglected with respect to the first term. This arrangement assures that the consecutive zones are shifted in phase by a fixed amount of $\pi$. These elements can be tailored in different configurations with binary amplitude, fractional phase subzone distribution, etc. the irradiance at the location of the focal point of the lens depends on the square of the total number of zones. This increase of the irradiance at the focus can be defined as a focalization efficiency [3] because it describes the comparative performance when the FZP is used versus the situation of not using it.

In a recent contribution [12], it has been shown how a polygonal FZP can be optimized to perform as close as possible to the circular FZP. The optimization depends on the number of sides of the polygon, $s$. The result of this optimization analysis is a regular polygon having an apothem value, $x_{m}$, given by the following relation 


$$
x_{m}=\frac{r_{m}}{\sqrt{1+\frac{\tan ^{2}(\pi / s)}{4}}} .
$$

The regular polygon substitutes the circumference as the perimeter defining the limits of the successive Fresnel zones. When the polygon is a square, $s=4$, the apothem is equal to $x_{m}=\frac{2}{\sqrt{5}} r_{m}$. The phase of each zone is calculated after adding together the phase of all the wavelets coming from the given zone and arriving to the focal point of its circular FZP version. The total amplitude for each zone, $A_{m}$, is given by

$$
A_{m}=\int_{-x_{m+1}}^{x_{m+1}} \int_{x_{m+1}}^{x_{m+1}} E(x, y) d x d y-\int_{-x_{m}}^{x_{m}} \int_{-x_{m}}^{x_{m}} E(x, y) d x d y,
$$

where the integration limits are equal for the two directions because our domain of integration is a square. The amplitude in the previous integral is given by

$$
E(x, y)=\frac{1}{r(x, y)}\left[\frac{1}{2}\left(1+\frac{f^{\prime}}{r(x, y)}\right)\right] \exp \left[-i \frac{2 \pi n}{\lambda_{o}} r(x, y)\right],
$$

being $r(x, y)$ the distance between the point $(x, y)$ at the FZP plane and the focus, $f^{\prime}$ is the focal distance, $n$ is the index of refraction of the medium after the FZP, and $\lambda_{0}$ is the wavelength in vacuum used in the design of the FZP. The phase of each square zone can be calculated as

$$
\Phi_{m}=\text { Phase }\left[A_{m}\right]
$$

The modulus of the complex amplitudes, $A_{m}$, and their phases, $\Phi_{m}$, are presented in Figure 1 as vectors associated to each zone. The length of the vector is the modulus of the corresponding amplitude, and the phase, $\Phi_{m}$, is represented by the orientation of the vector. 
This figure also includes the representation of the amplitudes for a circular FZP. The modulus of the amplitude of each zone can be easily evaluated numerically. A method to obtain the value of this modulus experimentally will be discussed in section 4 .

After evaluating the phase, $\Phi_{m}$, for each zone, we found that the relative phase shift between adjacent zones is constant and equal to $4 \pi / 5\left(144^{\circ}\right)$. Only the phase difference between the central zone and the following one is different and it is equal to $1.05 \pi\left(188.67^{\circ}\right)$. The circular FZP counterpart shows a phase difference of $\pi$ between adjacent zones. This already makes the SFZP a special element. The successive square zones have a collection of only five levels in phase (plus the phase value of the central zone). Therefore, if we want to add all the zones in phase we will only need to compensate these 5 phase-shifts and add them together with the central one. Taking this into account, it is possible to manufacture a square phase-mask with only these 6 phase levels (one for the center, and the other 5 as a repetitive pattern along the zones). In Figure 2 we show the phase levels of the SFZP as gray levels. Only 6 gray levels appear in the design. Besides the phase of the central zone, the phases follow a regular pattern every five zones. When calculating the irradiance at the focal point of the SFZP we have found that this irradiance increases linearly with the number of involved zones, instead of the quadratic dependence shown by the circular FZP. This is the price paid in terms of performance to get the benefit of managing a simple square geometry and the existence of only five phase steps along the element.

\section{Numerical simulation of the focusing capabilities of Square Fresnel Zone Plate}

An analytical solution for the irradiance distribution around the focus of this SFZP involves eqs. (3)-(5), and its development may obscure the simplicity of the SFZP behavior. As a consequence, we preferred to perform a numerical analysis to determine the focusing 
capabilities of this lens. For the numerical implementation, we have used a fast-Fouriertransform based direct-integration method which uses the Rayleigh-Sommerfeld approach [17]. The field $E(\xi, \eta, z)$ at the observation plane can be computed from the field just after the lens $E(x, y, 0)$ by means of the following integration

$$
E(\xi, \eta, z)=\int_{A} E(x, y, 0) \frac{\exp (i k r)}{2 \pi r} \frac{z}{r}\left(\frac{1}{r}-i k\right) d x d y
$$

where $(x, y)$ are the coordinates over the surface of the lens, $(\xi, \eta)$ are the coordinates at the observation plane, $z$ is the distance between the lens and the observation plane, $r=\sqrt{\xi^{2}+\eta^{2}+z^{2}}, k$ is the wavenumber, and $A$ is the integration area where the lens is defined. This scalar approach is valid as long as the features of the SFZP are larger than the wavelength. In addition, the proposed algorithm presents a quality parameter which indicates when the simulation is done properly.

The conditions for the simulations are similar to those of the experimental set-up that will be explained in detail in Section 4. A plane wave with wavelength $\lambda=632.8 \mathrm{~nm}$ arrives to the SFZP in normal incidence. For instance, we have used a lens with a focal $f^{\prime}=0.5 \mathrm{~m}$. The pixel size of the SFZP is $19 \mu \mathrm{m}$. For the simulations we have performed a re-sampling of the SFZP so that the pixel size in the propagation is similar to the pixel size of the CCD camera (6 $\mu \mathrm{m}$ ). The effects shown here are similar for other wavelengths and focals.

Firstly we are going to analyze the focusing properties of the SFZP and its dependence with the number of zones. In Figure 3 we show both the simulated and the experimental results. Now, in this section, we focus on the irradiance maps shown in the right column of this figure. The first and second rows correspond to the irradiance maps at the focus of two lenses having the same focal length but involving a different number of square Fresnel zones. The first row is for 12 zones and the second row is for 100 zones. Both maps are independently 
normalized to their maximum. We may see how the focus shrinks more efficiently as the number of zones grows. Also in this figure we have plotted the irradiance map obtained around the focus. It is easy to check how the irradiance is spread over a larger area. In addition to a bright spot at the center of the image we observe a cross-like shape at the focus for all the cases considered here. Also four small lobes at the diagonals are observed. The cross-like pattern has been observed previously [8],[9],[10],[11].

In Figure 4a the irradiance around the focus for a meridional plane $(x, z)$ containing the optical axis of the lens is shown for a SFZP with 12 zones. In Figure 4b the irradiance at the axis is plotted for SFZPs with different number of zones. We can see that the intensity at the maximum increases as the number of zones increases. On the other hand, the depth of focus decreases. When extending along the optical axis the calculation of the irradiance profile for a lens having 50 Fresnel zones we observe in Figure 4c a faint, but distinguishable secondary focus at approximately $z=f^{\prime} / 3$. This result is similar to that obtained with standard circular FZP and can be interpreted as a high diffraction order. In a standard circular zone plate, secondary focus are observed at distances $\pm f^{\prime} / 3, \pm f^{\prime} / 5, \ldots$, if the even zones are obscured. This feature comes from the dependence of the focal distance with the integer label of each non-obscured zone [1]. As the SFZP design tries to reproduce the performance of a standard FZP, multifocal performance is expected. In order to show how differently behave the square and circular FZP we show in Figure 5 two valuable comparisons. The plot on top represents the irradiance profiles along the direction of the $X$ (or $Y$ ) axis of the lens and also along a diagonal direction. The irradiance profile for a circular FZP has been also plotted. The calculation has been done for two lenses having 50 zones: one of the lens has circular Fresnel zones, and the other has square Fresnel zones. The irradiance has been normalized to the maximum for each one of the plots. We can see that the square Fresnel zone behaves quite differently along the $X$ (or $Y$ ) direction with respect to the profile along the diagonal. This is 
due to the cross-like diffraction pattern associated with the square symmetry of the diffractive element. This can be also inferred from the irradiance distribution shown in Figure 3. The circular FZP produces a sharper and narrower maximum than the square FZP. The plot at the bottom of this figure shows the behavior along the propagation axis. Also the circular FZP shows a narrower depth of focus than the square FZP.

\section{Experimental results}

A He-Ne laser ( $\lambda=632.8 \mathrm{~nm}$ ) is employed as a coherent light source impinging on a Spatial Light Modulator (SLM) that is in charge of the realization of the phase mask. The SLM is a Holoeye LCR-2500 reflective modulator, with 1024x768 pixels with 8 bits resolution each. It is addressed through the graphic board of the computer. The phase map is directly loaded on the SLM that acts as secondary monitor in the computer. It should be mentioned that the SLM inherently adds a non-negligible amount of astigmatism due to fabrication errors. We have adopted previous successful approaches used to solve these problems noticed in the same kind of SLM devices [18],[19],[20]. The phase of an astigmatic lens is added to the phase map of the Fresnel lens to compensate the astigmatism. The phase of the correction lens is

$$
\phi_{\text {elliptical }}=\frac{\pi}{\lambda}\left[\left(\frac{x \cos \theta+y \sin \theta}{\sqrt{f_{1}}}\right)^{2}+\left(\frac{-x \sin \theta+y \cos \theta}{\sqrt{f_{2}}}\right)^{2}\right] \text {, }
$$

where the parameters $f_{1}$ and $f_{2}$ are the Sturm foci and $\theta$ is the angle between the lens axes and the laboratory axes. These parameters are experimentally adjusted until no aberrations are observed resulting $f_{1}=14 \mathrm{~m}, f_{2}=8 \mathrm{~m}$, and $\theta=-167.7^{\circ}$. The actual phase map sent to the SLM is plotted in Figure 6 for a SFZP having 30 zones. 
As we may see in Figure 7, two lineal polarizers, P1 and P2, control the beam intensity and the polarization state of the emitted light. The Spatial Filter, SF, consists of a $40 \times$ microscope objective, conjugated with a $10 \mu \mathrm{m}$ pinhole. Setting the pinhole in the focal point of the microscope objective, we ensure a Gaussian-like beam illumination onto the SLM. The Collimating Lens, CL, is chosen in order to obtain the desired illumination field size, which is larger than the SLM. We use a 50/50 beam-splitter, BS, placed at $45^{\circ}$, so we can manage the SLM in the standard normal incidence configuration. The reflected light modulated by the device is redirected by means of the BS to a UI-1220-M camera, from U-Eye, with a pixel pitch of $6 \mu \mathrm{m}$. The camera travels along the optical axis, so as to make any measurements at any desired plane.

The calculated phase distribution for a given SFZP has been adapted to the operating specification of our reflective SLM (Model LCR-2500 from Holoeye). The main adaptation is related with the fit of the square Fresnel zones to the pixel size and period. The pixels are regularly spaced along a rectangular grid having the same period for the two orthogonal directions. This period is $19 \mu \mathrm{m}$. When calculating the square Fresnel zones, the limit of each zone typically falls at a given pixel. Then, this boundary pixel is shared by two adjacent square Fresnel zones. We have limited our design in order to avoid that three zones could share a single pixel. The phase associated to those boundary pixels is calculated as the weighted mean of the phases corresponding to the shared zones. The weights are given by the area of each zone over the pixel area. This also applies to the pixels along the diagonal of the square. This correction is a necessary step to take advantage of the flexibility in phase of the SLM so as to prove the performance of the proposed device.

Thus, in order to check the predictions arising from the Rayleigh-Sommerfeld integral computations, we have carried out measurements of the dependence of the maximum power at focus vs the number of zones, the results, along with the predictions from the simulation 
are shown in Figure 8. Practically, a collection of lenses has been projected onto the SLM. The set comprises lenses with a number of zones in the range $m=[5,100]$. They are characterized by the same focal distance, $f^{\prime}=0.5 \mathrm{~m}$. For each lens, a map of the intensity has been recorded at the focal plane. Five images were taken and averaged for each map to minimize noise. These averaged images are the ones used in the analysis. The gain of the CCD has been adjusted at a level that keeps the pixel under saturation for the maximum irradiance obtained with a SFZP with 100 zones. When representing the value of the maximum of the irradiance map vs. number of zones, in Figure 8. the curve shows a linear trend up to the $55^{\text {th }}$ zone. The boundary pixels for those zones after the $35^{\text {th }}$ are, at most, only 2 pixels apart. Actually, for the lenses tested in this contribution, the zone $51^{\text {st }}$ is the first to have the next boundary pixel (the one corresponding to the $52^{\text {nd }}$ ) in the next one. Therefore, we think this is one of the main reasons precluding the observation of the linear dependence for a larger number of zones. When increasing the number of zones further, the irradiance at focus increases at a slower rate in comparison to lenses with a low number of zones. A fit to a linear dependence $I=I_{0}+\eta m$ has been done in the first region. The parameters of the fit to the curve are $I_{0}=0.138 \pm 0.005$ and $\eta=0.0159 \pm 0.0003$ (the curve has been normalized to its maximum). The linear regression coefficient is $R=0.9612$. These results prove the linear behavior of the SFZP with the number of zones. The modulus of the amplitude of each zone can be obtained experimentally from a recursive method by computing the difference in irradiances. For any given zone this difference can be related with the sum of the modulus of the amplitudes of the previous zones, and with the amplitude itself of the current zone. When departing from the first zone, the iteration of this procedure makes possible this experimental evaluation. Unfortunately, the uncertainties related with our experimental set-up may mask the correct experimental evaluation of the modulus of the amplitude of each zone. 
In Figure 3 we compare the experimental irradiance with the numerical results obtained using the Rayleigh-Sommerfeld approach for different cases in and out of focus. It can be noticed the good agreement of the measured irradiance map with the results from the theory. It is significant the cross-like shape of the distribution at focus. The focus size is greater for a lens with $N=12$ than for $N=100$. The irradiance is redistributed in the focal plane, thus enlarging

the size of the focal spot and diminishing the power content in the area defined by it. Consequently, there is a smooth transition in the focusing capabilities of the diffractive elements as the number of zones grows up.

Likewise, it is worthwhile to analyze the out of focus performance of these lenses. Figure 3 also shows the irradiance of a 100 zones lens at the planes $\Pi_{a^{+}}$and $\Pi_{a-}$, where $a^{ \pm}=f^{\prime} \pm 1 \mathrm{~cm}$. The irradiance at these planes is not completely symmetric. This behavior has been observed for lenses with a different number of zones both numerically and experimentally.

\section{Conclusions}

In this work, we have analyzed the optical behavior of Square Fresnel Zones Plates. This kind of diffractive lens has only 6 phase levels, one for the central zone and 5 phase levels that are arranged as a regular pattern showing a constant phase difference between consecutive zones of $4 \pi / 5$. The price paid for this simple phase configuration is in terms of focusing efficiency. The irradiance at the focus increases linearly with the number of zones included in the design. The behavior of these lenses has been numerically proven. Properties such as the depth of focus and the intensity of the focus have been studied in terms on the number of zones using a numerical implementation of the RayleighSommerfeld approach. In addition, an experimental verification has been performed using 
a Spatial Light Modulator for implementing the designed Square Fresnel Zones Plates.

The experimental data are in very good agreement with the numerical results.

\section{Acknowledgements}

The authors are grateful to Prof. Eusebio Bernabéu. This work has been partially supported by project TEC2005-1882 and project CCG08-UCM/DPI-3952 of “Dirección General de Universidades e Investigación de la Consejería de Educación de la Comunidad de Madrid” y “Universidad Complutense de Madrid”. Salgado-Remacha acknowledges the economical support of "Ministerio de Educación y Ciencia” and the European Social Fund. 


\section{References}

[1] E. Hecht, Optics, Addisson Wesley, Reading, Mass, USA 2001.

[2] H. D. Hristov, Fresnel zones in wireless links, zone plate lenses and antennas, Artech House, Norwood, MA, USA, 2000.

[3] F. J. González. J. Alda, B. Illic, G. Boreman, Appl. Opt. 43 (2004) 6067-6073.

[4] J. Alda, J. M. Rico-García, J. M. López-Alonso, B. Lail, G. Boreman, Opt. Commun. 260 (2006) 454-461.

[5] U. Levy, N. Cohen, D. Mendlovic, Appl. Opt. 38 (1999) 5527-5532

[6] L. B. Lesem, P.M. Hirsch, J. A. Jordan, IBM J. Res. Dev. 13 (1969) 150-155.

[7] H. Zhang, H. Liu, Z. Lu, H. Zhang, Appl. Opt. 47 (2008) 4055-4060.

[8] J. M. Cuadrado, M. V. Perez, and C. Gomez-Reino, Appl. Opt. 26 (1987) 1527-1529.

[9] W. B. Hermannsfeldt, M.J. Lee, J.J. Spranza, and K. R. Trigger, Appl. Opt. 7 (1968) 9951005.

[10] Z. Jaroszewicz, A. Kolodziejczyk, A. Mira, R. Henao, and S. Bara, Opt. Express. 13 (2005) 918-925.

[11] J. M. Cuadrado, C. Gomez-Reino, and M. V. Perez, Opt. Acta 29 (1982) 717-723

[12] J. Alda, G. Boreman, Microwave Opt. Technol. Lett. 50 (2008) 536-541.

[13] L. J. Janicijevic, J. Opt. 13 (1982) 199-206.

[14] B. Zhang, D. Zhao, and S. Wang, Appl. Phys. Lett. 91 (2007) 021108.

[15] I. V. Minin, O. V. Minin, A. Petosa, S. Thirakuone, Microwave Opt. Technol. Lett. 49 (2007) 276-278.

[16] J. Ginn, B. Lail, J. Alda, G. Boreman, Opt. Lett. 33 (2008) 779-781.

[17] F. Shen, and A. Wang, Appl. Opt. 45 (2006) 1102-1110.

[18] E. Pleguezuelos, J. Andilla, A. Carnicer, E. Martín-Badosa, S. Vallmitjana, and M. Montes-Usategui, Proc of SPIE 6326 (2006) 63262Q.

[19] J. Otón, P. Ambs, M. S. Millán, and E. Pérez-Cabré., Appl. Opt. 46 (2007) 5667-5679. 
[20] M.L. Cordero, D.R. Burnham, C.N. Baroud, D. McGloin, Appl. Phys. Lett. 93 (2008) 034107. 


\section{Figure captions}

Figure 1. Representation of the complex amplitude for each Fresnel zone for the square polygonal shape (s=4). The thin arrows are for the circular FZP and the bold ones are for the square FZP. The relative orientation of these amplitudes represents the phase of each one. We may see that they are aligned in five different directions following a pattern.

Figure 2: Map of the phase of the square FZP having 21 zones. The diameter of the SFZP is $4.598 \mathrm{~mm}$. This element is designed for $\lambda_{0}=632.8 \mathrm{~nm}$, and having a focal length of $0.5 \mathrm{~m}$.

The levels are given in degrees and correspond with a consecutive phase shift of $4 \pi / 5=144^{\circ}$. These five levels with this phase interval produce the represented levels of $0^{\circ}$, $72^{\circ}, 144^{\circ}, 216^{\circ}$, and $288^{\circ}$. The level at 188.67 corresponds with the central zone. The $0^{\circ}$ level is set for the second zone (the following to the central one).

Figure 3. Comparison between the irradiance maps obtained from the measurements (left columns) and the simulations (right columns) having the same optical and geometrical parameters for each row. All the lenses are having a focal length of $f^{\prime}=0.5 \mathrm{~m}$, for a wavelength of $632.8 \mathrm{~nm}$. The first row is for a lens having 12 Fresnel zones. The second, third and fourth rows are for a lens with 100 Fresnel zones at the focal plane, before ( $f$ '- 1 $\mathrm{cm})$, and after $\left(f^{\prime}+1 \mathrm{~cm}\right)$ the focal plane respectively. The irradiance maps are plotted within a square of $600 \times 600 \mu \mathrm{m}$. Each map is normalized to its maximum value.

Figure 4. a) Map of the irradiance for a meridional plane containing the optical axis. b) Irradiance profile along the axis of propagation for several lenses with $f^{\prime}=0.5 \mathrm{~m}$, and several number of zones $N=20$ (the lowest and widest), 30, 40, 50, 60, 70, 80, 90 and 100 (the largest and sharpest). As expected, the depth of focus shrinks as the number of zones increases. c) Normalized irradiance along the z-axis. The $\mathrm{z}$ axis is normalized to the focal 
distance of the lens. The inset shows a zoom on the secondary focus at approximately $z \approx f^{\prime} / 3$.

Figure 5. Irradiance profiles for the square FZP and another circular FZP having the same number of zones (50 zones). This element is designed for $\lambda_{0}=632.8 \mathrm{~nm}$, and having a focal length of $0.5 \mathrm{~m}$. The figure at the top represents the profiles for the square FZP along the Xaxis (or Y-axis) as a solid line, and along the diagonal as a dotted line. The irradiance profile for the circular FZP is plotted as a dashed line. At the bottom we plot the irradiance profile along the axis of propagation (Z-axis) for the square (solid line) and circular FZP (dashed line).

Figure 6. Elliptical phase used to compensate the astigmatism due to the SLM plus a Fresnel zone plate of 30 zones with a diameter equal to $5.5 \mathrm{~mm}$. The gray levels range from 0 up to 255.

Figure 7. Schematic view of the experimental set up.

Figure 8. Dependence of the maximum irradiance vs the number of square zones. The theoretical computation is plotted as a solid line. The experimental results are given as solid circles. Also a linear fitting vs number of zones is shown as a dashed line. The linear fit involves lenses from the $5^{\text {th }}$ to the $55^{\text {th }}$ zone.. In the inset we show the complete set of measurement up to 100 zones. The trend in the measured data is linear up to the 55th zone approximately, where the intensity grows up in a slower fashion. All the irradiance values have been normalized to the value at $55^{\text {th }}$. 
Figure 1

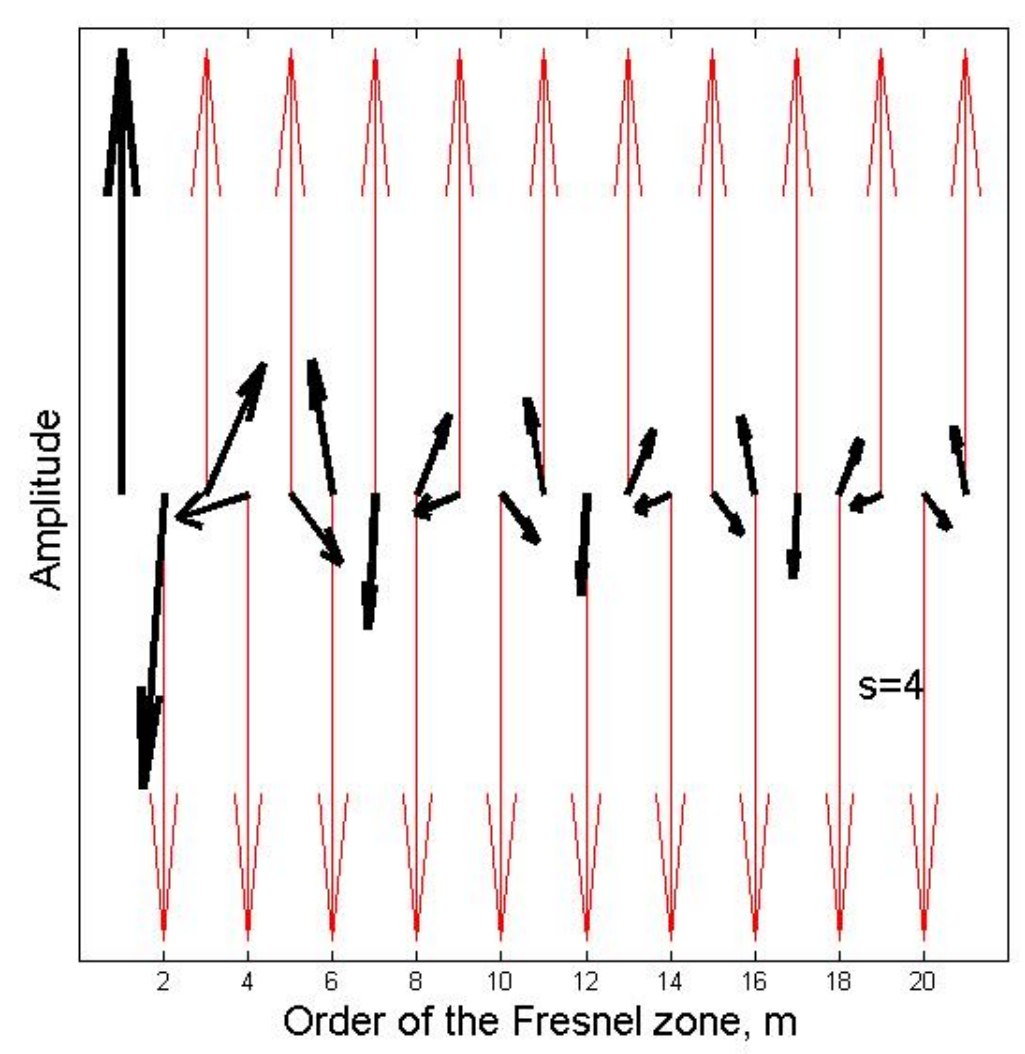


Figure 2

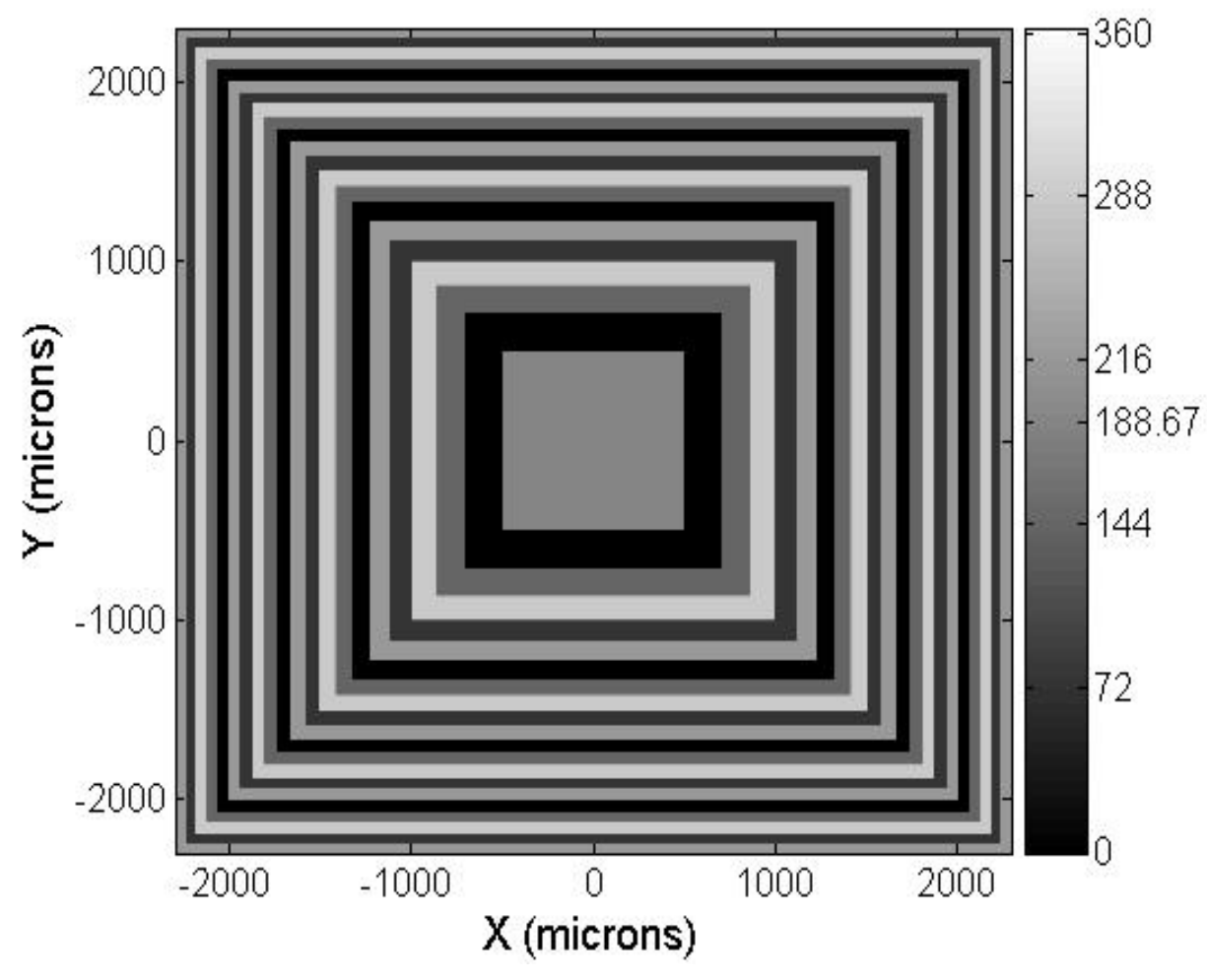


Figure 3

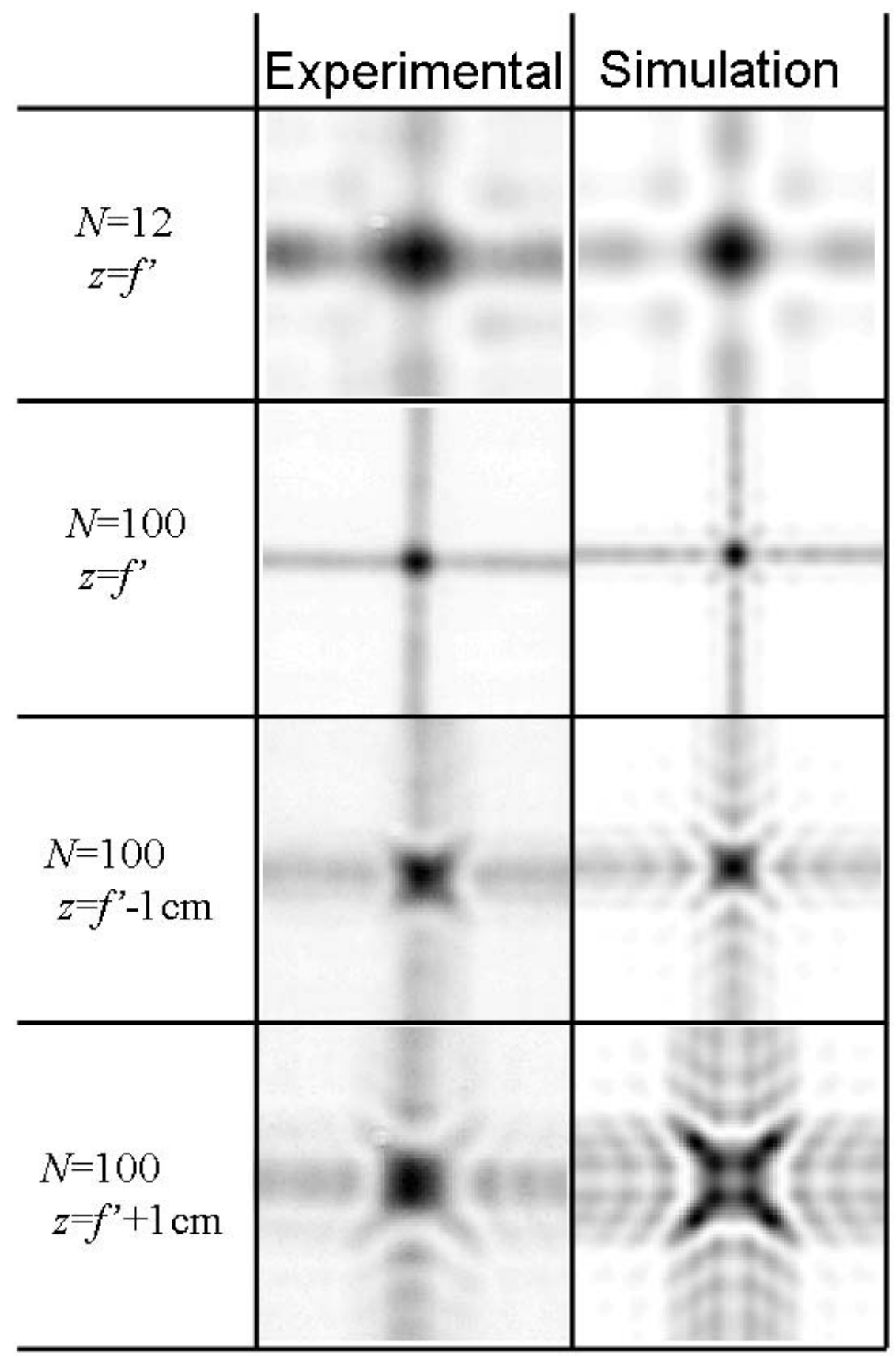


J. Alda et al. "Diffractive Performance of Square Fresnel Zone Plates"

Figure 4
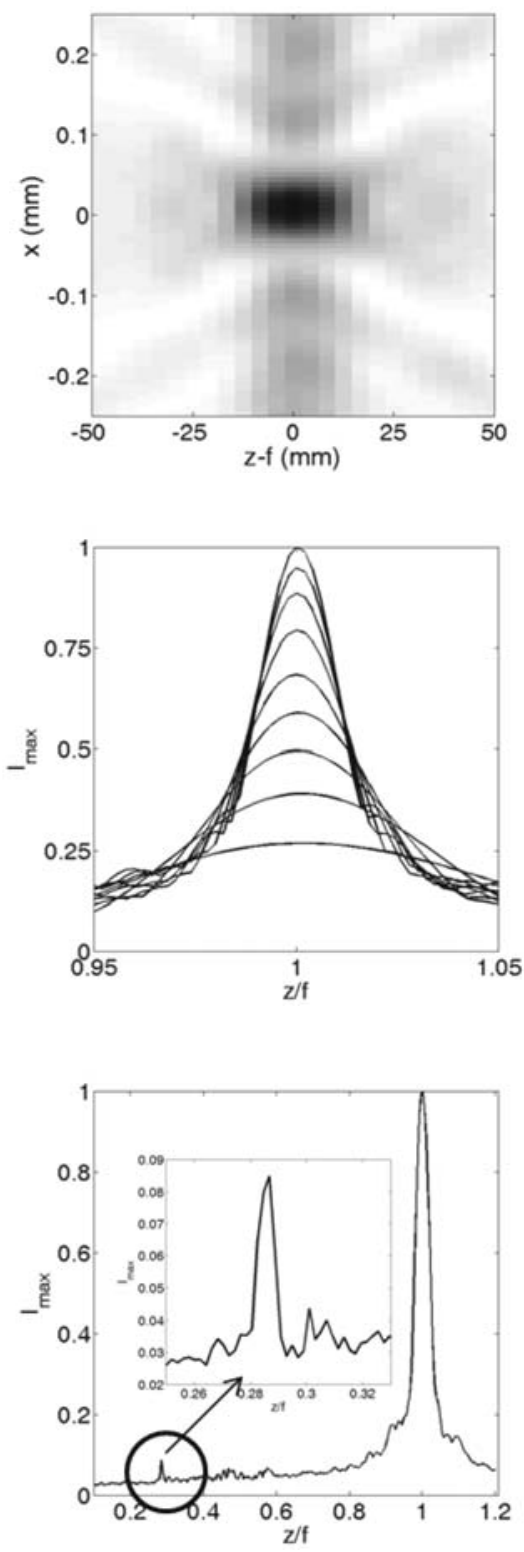
Figure 5
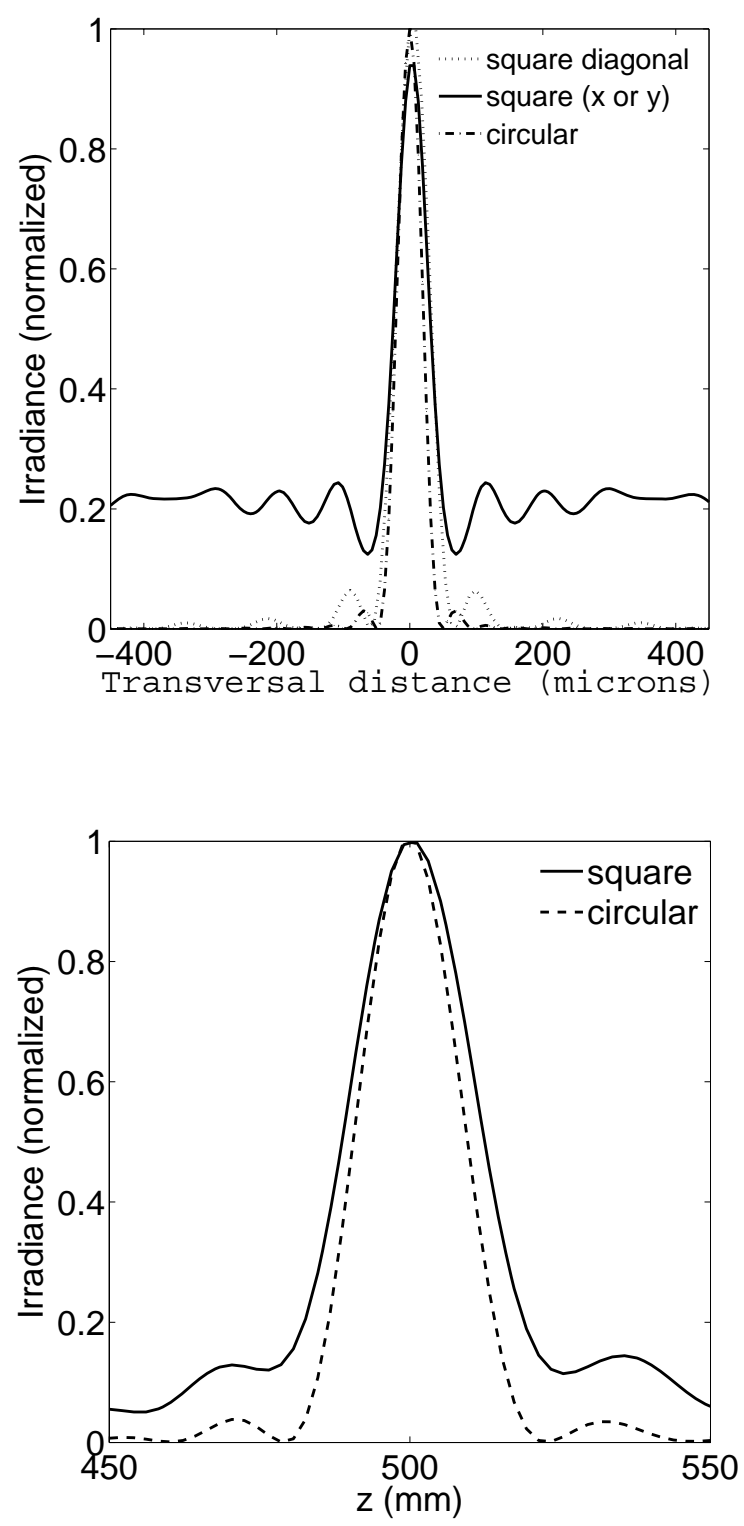


\section{Figure 6}

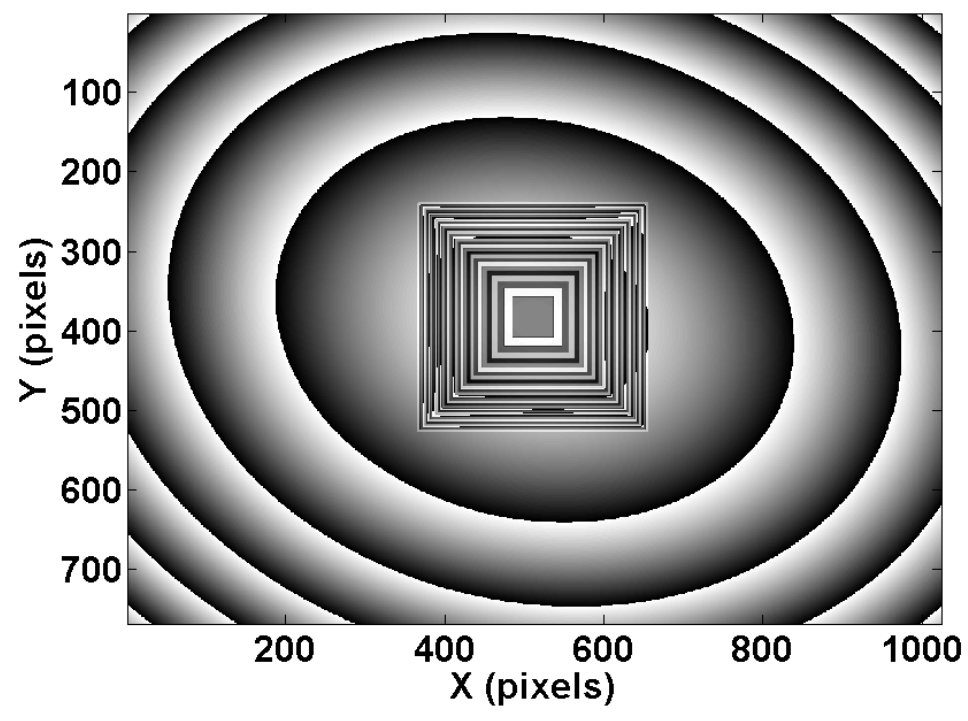


Figure 7.

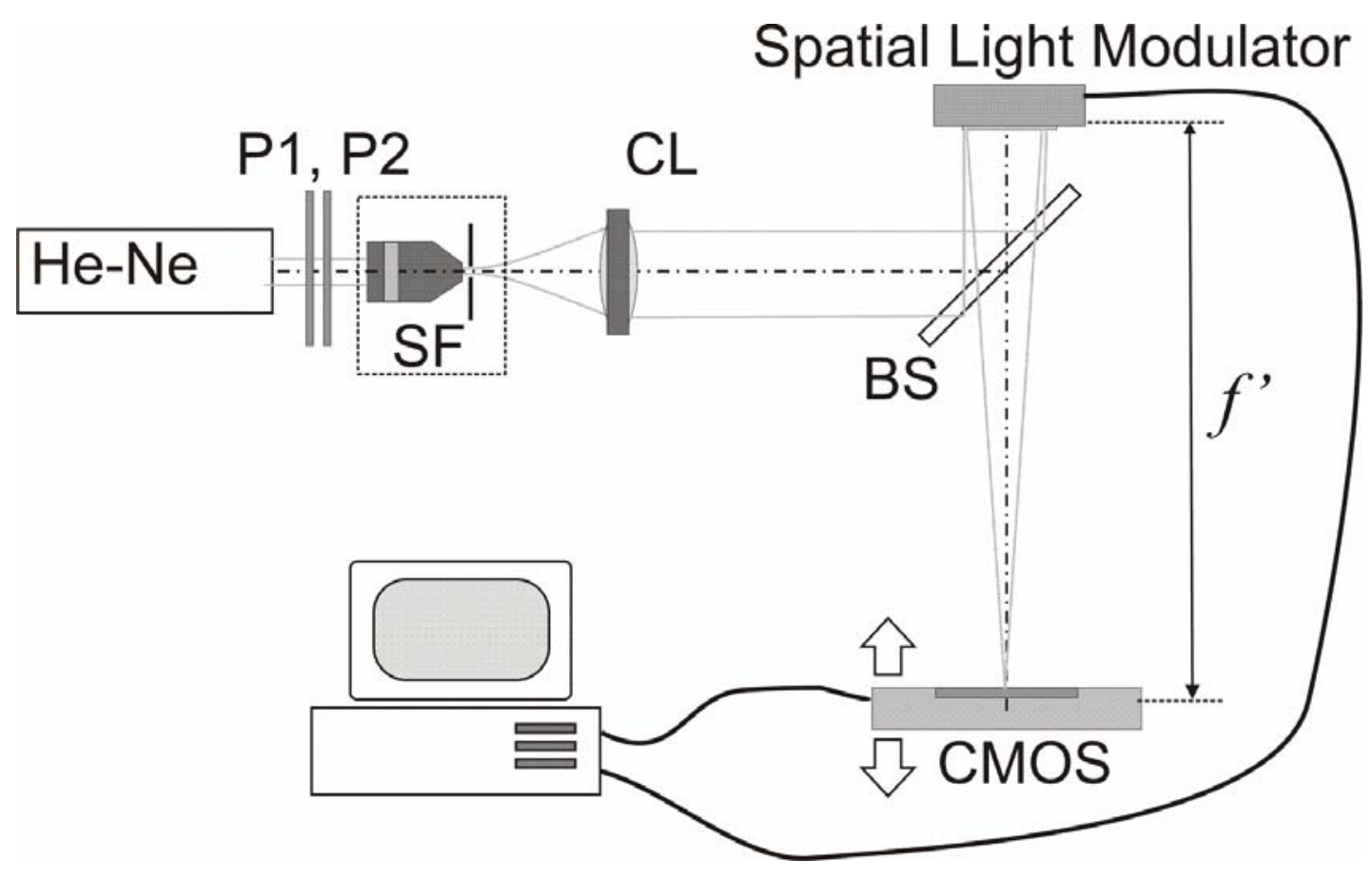


Figure 8.

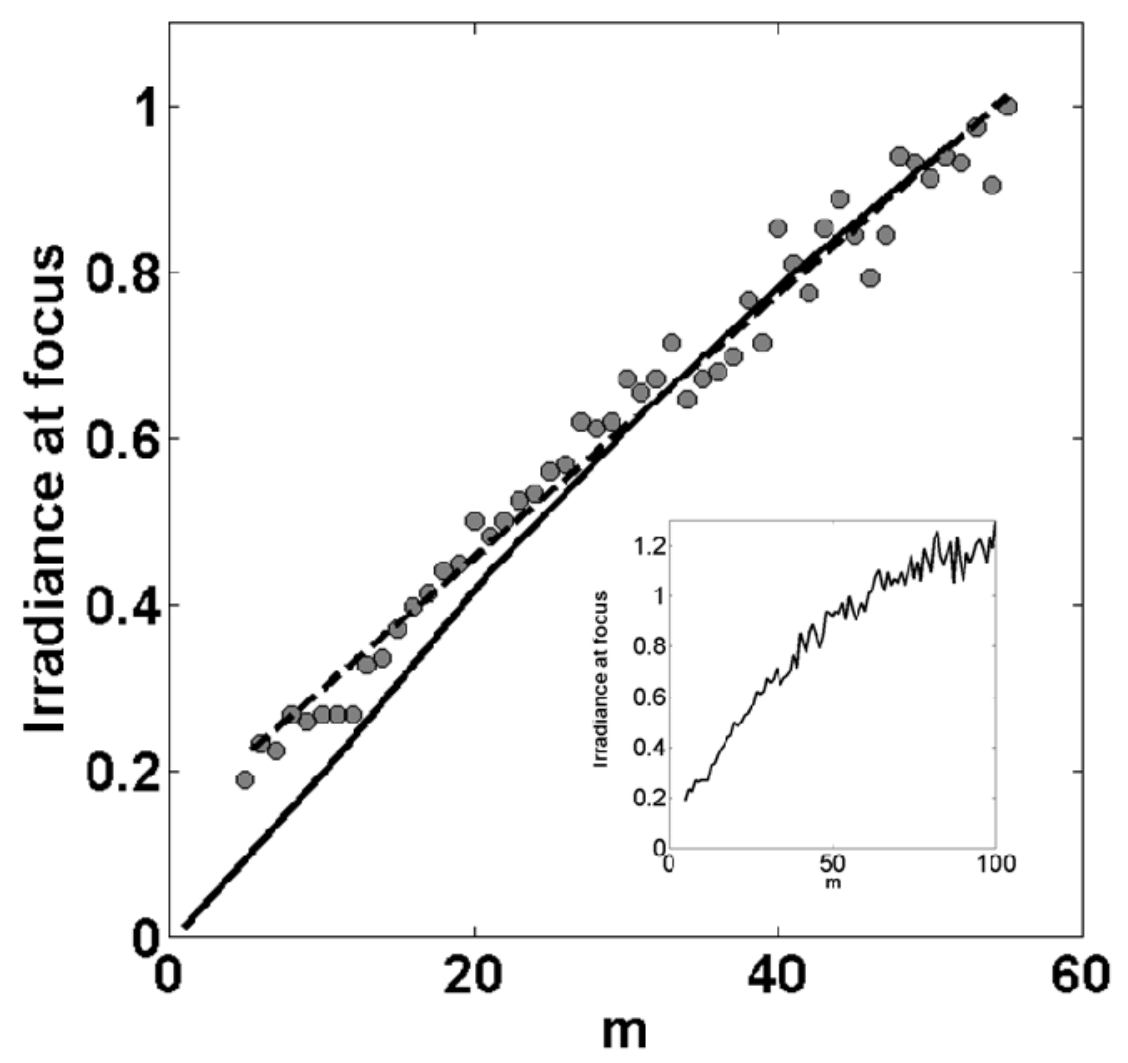

